\title{
The changes of oral health-related quality of life and satisfaction after surgery-first orthognathic approach: a longitudinal prospective study
}

\author{
Shengbin Huang ${ }^{1}$, Weiting Chen ${ }^{2}$, Zhenyu $\mathrm{Ni}^{2}$ and Yu Zhou ${ }^{2^{*}}$
}

\begin{abstract}
Background: To our best knowledge, there was little research to assess the changes of quality of life and satisfaction after orthognathic in one trial. The aim of this study was to evaluate the changes of oral health related quality of life and satisfaction between surgery-first and orthodontic-first orthognathic surgery.

Methods: Fifty Chinese orthognathic adluts patients completed two questionnaires: the Dental Impact on Daily Living questionnaire for assessment of his/her satisfaction and 14-item Oral Health Impact Profile for assessment of patient's quality of life. The subjects completed six sets of interviews and clinical evaluations at before treatment; 1 month after surgery (surgery-first); 6 months after treatment; 12 months after treatment; and 18 month after treatment ; the finished treatment. The pre and post surgical orthodontic period was also recorded. Chi square tests and repeated-measures analysis of variance (ANOVA) were used to compare categorical variables and measure results. All analyses were carried out used Stata software.

Results: The quality of life was significant improved when finished treatment and the amounts of change did not show any significant difference in each domain and at 1, 6, 12 month after orthognathic surgery between two groups. However, in orthodontic-first group, the quality of life was deteriorated before orthognathic surgery. In surgery-first group, the quality of life was immediately improved which lead to better satisfaction.

Conclusions: Although the quality of life scores was no significant difference between two groups, surgery-first treatment could significant reduce treatment during and no deterioration stage of quality of life score which lead to better satisfactory compare to orthodontic-first group. However, some of limitations we need take caution. In future we still need conduct more study to assess the influence of surgery-first method on quality of life.
\end{abstract}

Keyword: Quality of life, Orthognathic surgery, Surgery-first, Orthodontic-first

\section{Background}

Dentofacial deformities are deformities that affect primarily the jaws and the dentition; therefore, they are extremely prominent, are not easily disguised, and affect one's quality of life immensely $[1,2]$. Orthognathic surgery aims to correct these deformities via various osteotomies to achieve a desirable end result. Results deemed to be satisfactory from a clinician's aspect may

\footnotetext{
* Correspondence: wzmczy@126.com

${ }^{2}$ Department of Orthodontics, Hospital of Stomatology, Wenzhou Medical

University, 113 West College Road, 325000 Wenzhou, China

Full list of author information is available at the end of the article
}

not be so from the patient's aspect, because studies have shown that in patients with facial deformity, there is a close relationship between patient satisfaction and psychosocial functioning [3-6]. In recent years, research on quality of life assessment has been on the rise, and, more importantly, the area of focus has widened with greater emphasis placed on social well-being rather than disease mortality, tumor growth, etc., providing muchneglected subjective views of the treatment outcomes [7]. With increased relevance of health-related quality of life (HRQoL), it is now recognized that quality of life 
(QoL) assessment is a key outcome measure in the management of dentofacial deformities [8-11].

However, the traditional orthognathic surgery treatment which included preoperative orthodontic treatment, orthognathic surgery, and postoperative orthodontic treatment have several disadvantage, such as long treatment during, worsen facial profile and dental function before received orthognathic surgery. Recently, a new surgery method was introduced to overcome the disadvantages of conventional surgical-orthodontic treatment procedures named surgery-first approach (SFA). Because this surgery did not need pre-operative orthodontic treatment, it can significant reduce total treatment time and improve facial profile immediately which may contribute to improving the satisfaction and quality of life in orthognathic surgerypatients [12-15].

Previous studies had showed that orthognathic surgery could improve dental esthetic and quality of life after treatment [16-18]. A recently systematic review [19] also showed that orthognathic surgery had a positive impact on the patient's facial appearance and oral function and found social advantages such as improved self confidence. However, the previous studies were concerning the changes of quality of life after traditional surgical-orthodontic treatment, it is also necessary to assess the influence of different surgery time on the quality of life. In additional, there was little research to investigate the improving patients' satisfaction after orthognathic surgery.

Thus, the aim of this study was to evaluate the changes of oral health related quality of life and satisfaction between surgery-first and orthodontic-first orthognathic surgery patients.

\section{Methods}

\section{Ethical}

Ethical approval was obtained from the Ethics Committee of the stomatology hospital of the WenZhou Medical University. (Ethics approval number: 201506352541) The participants were informed about the trail and given written consent.

\section{Participants}

A total of 50 patients who will receive orthognathic surgery at the Department of Orthodontic at the Stomatology Hospital of WenZhou Medical University were included in this study. The sample divided into 2 groups: the surgery-first group (female 12, male13; 24.2 \pm 5.8 years) and the orthodontic-first group (female 13, male $12 ; 25.2 \pm 4.2$ years).

The inclusion criteria were as follows: 1 ) severe Class III malocclusion need received orthognathic treatment; 2) patients receive either surgery-first or orthodonticfirst orthognathic treatment plan;3) orthognathic surgery consisted purely of bilateral sagittal split ramus osteotomy (BSSRO) to resolve mandibular setback. Subjects were excluded from the study if they had cleft lip and/or palate or other craniofacial anomaly; were taking medications; or had undergone previous orthognathic treatment.

Orthodontic treatment was carried out by one clinicians, and the surgical procedures also by one maxillofacial surgeons who familiar with orthodontist.

\section{Instruments and measures}

The patients were given two questionnaires, the Dental Impact on Daily Living (DIDL) questionnaire for assessment of his/her satisfaction after treatment and OHIP14 for assessment of patient's quality of life.

During the interviews, we collected the baseline information of patients such as: gender, age, and socialeconomic status before treatment. For assessing the patient's satisfaction after treatment, the DIDL questionnaire has 36 items that are placed in five major categories and tackles five major dimensions of dental satisfaction, namely appearance, pain, oral comfort, general performance, and chewing and eating (Appendix 1), was used. The DIDL scale measures the effect and the proportional importance of each dimension to the patient. The scale has a score from 0 to 10 to show the relative importance of each dimension to the patients [20].

The Chinese version of the OHIP-14 which has shown good psychometric properties was used to assess the changes of oral health-related quality of life. The Chinese Version of the OHIP-14 including seven domains: functional limitation, physical pain, psychological discomfort, physical disability, psychological disability, social disability, and any handicaps. Each item was scored on a 5-point scale to rate the OHQOL. The higher scores indicating poorer OHQoL.

The subjects completed six sets of interviews and clinical evaluations at before treatment (T1); 1 month after surgery (T2); 6 months after treatment (T3); 12 months after treatment (T4); and 18 month after treatment (T5); the finished treatment (T6). The pre and post surgical orthodontic period was also recorded.

\section{Data analysis}

Chi square tests were used to compare categorical variables. Repeated-measures analysis of variance (ANOVA) was performed to statistically compare the measurements at each time to the baseline values (T1) in each group. ANOVA determined whether there were any significant overall differences among the groups at each time. Additionally, to identify the significance in each pair of groups, multiple comparison analysis was also performed for the time when the significant difference was noticed. One-way ANOVA and multiple comparison 
analysis were performed to compare the amount of treatment during between two groups.

All analyses were carried out used Stata software (version 11.2; StataCorp, College Station, Tex). Significance levels were established at 0.05 .

\section{Results}

There was no significant difference between baseline between the surgery-first group and orthodontic-first group, see Table 1.

The mean treatment time was $16.6+2.4$ month in surgery-first group and $25.3+2.4$ month in orthodonticfirst group. Treatment time was significantly longer in the orthodontic-first group compared to the surgery-first group.

Satisfaction with the DIDL questionnaire was shown in Table 2. There was no significant difference between two groups in each domain. However, the scores were relatively lower in surgery-first group than orthodontic-first group, though this difference did not reach a significant level. This result indicated that surgery-first may acquire better satisfaction compared to orthodontic-first group.

Quality of life level in surgery-first group was significantly improved after 1 month surgery (T2) when compared with baseline $(P<0.000)$. However, no statistical differences were observed between T4 (after 12 month ) and T6 (end of treatment). In the orthodontic-first group, the quality of life was deteriorated until T3 (after 6 month treatment) though the difference was not significant. Following orthognathic surgery, a signifcant improved of quality of life levels was observed $(P<0.05)$. The changes in the quality of life levels are shown in detail in Tables 3 and 4.

Table 1 Demographic and clinical characteristics of surgery-first group and orthodontic-first group subjects

\begin{tabular}{|c|c|c|c|}
\hline & $\begin{array}{l}\text { Surgery-first } \\
\text { group }\end{array}$ & $\begin{array}{l}\text { Orthodontic-first } \\
\text { group }\end{array}$ & $\begin{array}{l}P \\
\text { value }\end{array}$ \\
\hline & $\begin{array}{l}\mathrm{n}(\text { mean } \pm \mathrm{SD} \\
\text { or } \%)\end{array}$ & $\begin{array}{l}\mathrm{n}(\text { mean } \pm \mathrm{SD} \\
\text { or \%) }\end{array}$ & \\
\hline \multicolumn{4}{|l|}{ Sex } \\
\hline Female & $12(48)$ & $13(52)$ & \\
\hline Male & $13(52)$ & $12(48)$ & NS \\
\hline \multicolumn{4}{|l|}{ Age } \\
\hline $18-25$ & 5 & 4 & \\
\hline $25-30$ & 15 & 15 & \\
\hline $30-35$ & 5 & 6 & NS \\
\hline \multicolumn{4}{|c|}{$\begin{array}{l}\text { Social-economic } \\
\text { class }\end{array}$} \\
\hline (high) & $20(51)$ & 19(49) & \\
\hline (low) & $5(45)$ & $6(55)$ & NS \\
\hline
\end{tabular}

Though the quality of life scores were lower in surgery-first group, the difference did not come to a significant level between two groups at 1 month, 6 month and 12 month after received orhognhic surgery. The group comparisons are shown in Table 5.

\section{Discussion}

This is the first study to evaluate patient's satisfaction, treatment duration, and quality of life changes between SFA and conventional orthognathic method in Chinese, and we found some interesting results.

One of the most highlighted benefits of SFA is the reduction in treatment duration. The reason may be that, after surgery, orthodontic tooth movement can be easily achieved because the teeth are usually not occluded. Recently, a systematic review evaluated a few retrospective cohort studies and a larger number of case reports treated with SFA [21]. The results showed that the majority of cases were treated under a year. This agrees with our findings which show an average treatment duration of 10.5 months. This is a clear advantage over the conventional approach where treatment times have been reported in the realm of 7-12 months [22-29]. The different total treatment times for SFA depend on the severity of individual dentoskeletal problems,techniques of surgery, orthodontic mechanics, cooperation and biological response as well as desired results for each patient.

Assessment of patient satisfaction with their dentition after orthodontic treatment was carried out using the DIDL questionnaire. The DIDL questionnaire is a reliable, valid, and comprehensive test to measure patient satisfaction and effect of dental disease on patient daily living $[30,31]$. The test has shown the ability to assess satisfaction with different aspects of oral cavity and dental status, and for these reasons, it was selected for this study. Orthodontic problems can affect many aspects of dental esthetics and function, and these aspects are well covered by the DIDL test.

Our results revealed that satisfaction after orthognathic surgery was high.

A total of 80 and $72 \%$ of all patients rated postoperative outcomes after surgery in two group, respectively. There was no significant difference between two groups in each domain. However, the scores were relatively lower in surgery-first group than orthodontic-first group, though this difference did not reach a significant level. This result indicated that surgery-first may acquire better satisfaction compared to orthodontic-first group. The high patient satisfaction in SFA group may due to immediate improvement of facial profile at the beginning of the treatment14,29-30. The high satisfaction rate was in accordance with that reported in previous studies, which 
Table 2 Frequency of Individual Satisfaction Dimensions in the Study Population

\begin{tabular}{|c|c|c|c|c|c|c|c|}
\hline \multirow[t]{2}{*}{ Dimension } & \multicolumn{3}{|c|}{ Surgery-first group } & \multicolumn{3}{|c|}{ Orthodontic-first group } & \multirow[t]{2}{*}{$\frac{P}{P}$} \\
\hline & Dissatisfied & Relatively Satisfied & Satisfied & Dissatisfied & Relatively Satisfied & Satisfied & \\
\hline Appearance & $0(\%)$ & $1(4)$ & $24(96)$ & 0 & $2(8)$ & $23(92)$ & NS \\
\hline Pain & $1(4)$ & $7(28)$ & $17(68)$ & $2(8)$ & $6(24)$ & $14(68)$ & NS \\
\hline Oral comfort & $3(12)$ & $4(16)$ & $18(72)$ & $2(8)$ & $7(28)$ & $16(64)$ & NS \\
\hline General performance & $0(0)$ & 2(8) & $23(92)$ & $0(0)$ & $3(12)$ & $22(88)$ & NS \\
\hline Eating and chewing & $1(4)$ & $2(8)$ & $22(88)$ & $2(8)$ & $2(8)$ & $21(84)$ & NS \\
\hline Total & $1(4)$ & $4(16)$ & 20(80) & $2(8)$ & $5(20)$ & $18(72)$ & NS \\
\hline
\end{tabular}

NS Non-significant

Table 3 Comparison of quality of life scores at before treatment (T1); 1 month after surgery (T2); 6 months after treatment (T3); 12 months after treatment (T4); and 18 month after treatment (T5); the finished treatment(T6) with T1 (before treatment) in two group

\begin{tabular}{|c|c|c|c|c|c|c|c|c|c|c|c|c|}
\hline Varies & Group & T1 & $\mathrm{T} 2$ & $\mathrm{P}(\mathrm{T} 2-\mathrm{T} 1)$ & T3 & $\mathrm{P}(\mathrm{T} 3-\mathrm{T} 1)$ & T4 & $\mathrm{P}(\mathrm{T} 4-\mathrm{T} 1)$ & T5 & $\mathrm{P}(\mathrm{T} 5-\mathrm{T} 1)$ & T6 & $P($ T6-T1) \\
\hline \multirow[t]{4}{*}{ functional limitation } & \multirow[t]{2}{*}{ Surgery-first group } & 8.42 & 5.22 & \multirow[t]{2}{*}{$0.034^{*}$} & 2.39 & \multirow[t]{2}{*}{$0.000^{* * *}$} & 1.99 & \multirow[t]{2}{*}{$0.000^{* * *}$} & 1.98 & \multirow[t]{2}{*}{$0.000^{* * *}$} & 1.89 & \multirow[t]{2}{*}{$0.000^{* * *}$} \\
\hline & & $(2.79)$ & $(1.88)$ & & $(1.24)$ & & $(1.76)$ & & $(1.68)$ & & $(1.26)$ & \\
\hline & \multirow[t]{2}{*}{ Orthodontic-first group } & 8.45 & 8.30 & \multirow[t]{2}{*}{0.867} & 8.79 & \multirow[t]{2}{*}{0.758} & 5.42 & \multirow[t]{2}{*}{$0.000^{* * *}$} & 1.98 & \multirow[t]{2}{*}{$0.000^{* * *}$} & 1.92 & \multirow[t]{2}{*}{$0.000^{* * *}$} \\
\hline & & (2.99) & $(2.56)$ & & $(1.23)$ & & $(1.98)$ & & (1.68) & & $(1.56)$ & \\
\hline \multirow[t]{4}{*}{ physical pain } & \multirow[t]{2}{*}{ Surgery-first group } & 5.26 & 3.22 & \multirow[t]{2}{*}{$0.045^{*}$} & 2.34 & \multirow[t]{2}{*}{$0.009^{* *}$} & 1.59 & \multirow[t]{2}{*}{$0.000^{* * *}$} & 1.56 & \multirow[t]{2}{*}{$0.000^{* * *}$} & 1.34 & \multirow[t]{2}{*}{$0.000^{* * *}$} \\
\hline & & $(1.85)$ & $(1.55)$ & & $(1.24)$ & & $(0.67)$ & & $(0.51)$ & & $(0.89)$ & \\
\hline & \multirow[t]{2}{*}{ Orthodontic-first group } & 5.46 & 5.30 & \multirow[t]{2}{*}{0.920} & 6.02 & \multirow[t]{2}{*}{0.052} & 3.51 & \multirow[t]{2}{*}{$0.000^{* * *}$} & 1.89 & \multirow[t]{2}{*}{$0.000^{* * *}$} & 1.75 & \multirow[t]{2}{*}{$0.000^{* * *}$} \\
\hline & & $(1.61)$ & $(1.69)$ & & $(2.36)$ & & $(1.72)$ & & $(1.28)$ & & $(1.22)$ & \\
\hline psychological discomfort & Surgery-first group & 6.12 & 4.29 & $0.048^{*}$ & 2.22 & $0.000^{* * *}$ & 0.56 & $0.000^{* * *}$ & 0.52 & $0.000^{* * *}$ & 0.45 & $0.000^{* * *}$ \\
\hline & & $(2.12)$ & $(1.22)$ & & $(1.12)$ & & $(1.10)$ & & $(0.18)$ & & $(0.13)$ & \\
\hline & Orthodontic-first group & 6.25 & 6.52 & 0.685 & 6.56 & 0.658 & 4.82 & $0.000^{* * *}$ & 2.82 & $0.000^{* * *}$ & 0.92 & $0.000^{* * *}$ \\
\hline & & $(2.09)$ & $(2.23)$ & & $(2.85)$ & & $(2.29)$ & & $(1.31)$ & & $(1.26)$ & \\
\hline physical disability & Surgery-first group & 6.29 & 4.15 & $0.045^{*}$ & 1.64 & $0.000^{* * *}$ & 1.34 & $0.000^{* * *}$ & 0.12 & $0.000^{* * *}$ & 0.10 & $0.000^{* * *}$ \\
\hline & & $(2.64)$ & $(1.72)$ & & $(1.08)$ & & $(1.22)$ & & $(0.11)$ & & $(0.03)$ & \\
\hline & Orthodontic-first group & 6.39 & 6.56 & 0.687 & 6.85 & 0.785 & 4.42 & $0.000^{* * *}$ & 2.41 & $0.000^{* * *}$ & $1.49(0.91)$ & $0.000^{* * *}$ \\
\hline & & $(2.72)$ & $(2.67)$ & & $(2.75)$ & & $(1.75)$ & & (1.13) & & & \\
\hline psychological disability & Surgery-first group & 6.54 & 3.28 & $0.007^{* *}$ & 1.64 & $0.000^{* * *}$ & 0.42 & $0.000^{* * *}$ & 0.20 & $0.000^{* * *}$ & 0.15 & $0.000^{* * *}$ \\
\hline & & $(2.52)$ & $(1.76)$ & & $(1.08)$ & & $(0.21)$ & & $(0.12)$ & & $(0.12)$ & \\
\hline & Orthodontic-first group & 6.72 & 6.79 & 0.920 & 6.76 & 0.925 & 3.72 & $0.000^{* * *}$ & 1.83 & $0.000^{* * *}$ & 0.88 & $0.000^{* * *}$ \\
\hline & & $(2.42)$ & $(3.41)$ & & $(3.06)$ & & $(1.58)$ & & (1.15) & & $(1.13)$ & \\
\hline social disability & Surgery-first group & 6.03 & 4.26 & 0.042 & 1.24 & $0.000^{* * *}$ & 0.62 & $0.000^{* * *}$ & 0.19 & $0.000^{* * *}$ & 0.15 & $0.000^{* * *}$ \\
\hline & & $(2.32)$ & $(1.98)$ & & $(1.08)$ & & $(0.35)$ & & $(0.22)$ & & $(0.15)$ & \\
\hline & Orthodontic-first group & 6.12 & 6.86 & 0.865 & 6.89 & 0.675 & 4.52 & $0.000^{* * *}$ & 1.64 & $0.000^{* * *}$ & 0.98 & $0.000^{* * *}$ \\
\hline & & $(2.68)$ & $(2.88)$ & & $(2.29)$ & & $(2.56)$ & & (1.18) & & $(1.12)$ & \\
\hline handicaps & Surgery-first group & 3.24 & 2.18 & 0.035 & 1.52 & $0.004^{* *}$ & 0.38 & $0.000^{* * *}$ & 0.16 & $0.000^{* * *}$ & 0.12 & $0.000^{* * *}$ \\
\hline & & $(1.18)$ & $(1.81)$ & & $(1.75)$ & & $(0.26)$ & & $(0.12)$ & & $(0.12)$ & \\
\hline & Orthodontic-first group & 5.66 & 6.34 & 0.901 & 6.61 & 0.921 & 2.42 & $0.000^{* * *}$ & 1.94 & $0.000^{* * *}$ & 0.78 & $0.000^{* * *}$ \\
\hline & & $(2.81)$ & $(1.85)$ & & $(2.72)$ & & $(2.12)$ & & (1.36) & & $(1.12)$ & \\
\hline Total scores & Surgery-first group & 38.68 & 27.72 & $0.031^{*}$ & 13.94 & $0.000^{* * *}$ & 6.90 & $0.000^{* * *}$ & 4.11 & $0.000^{* * *}$ & 3.89 & $0.000^{* * *}$ \\
\hline & & $(4.35)$ & $(3.26)$ & & $(2.13)$ & & $(1.39)$ & & $(0.49)$ & & $(1.02)$ & \\
\hline & Orthodontic-first group & 39.55 & 41.67 & 0.654 & 48.48 & 0.124 & 28.86 & $0.000^{* * *}$ & 15.61 & $0.000^{* * *}$ & 8.68 & $0.000^{* * *}$ \\
\hline & & $(4.15)$ & $(4.14)$ & & $(3.91)$ & & (3.83) & & (2.49) & & $(1.65)$ & \\
\hline
\end{tabular}


Table 4 Statistical evaluation of quality of life scores between the surgery-first group and the orthodontic-first group at 1 month after surgery (T2); 6 months after treatment (T3); 12 months after treatment (T4); and 18 month after treatment (T5); the finished treatment (T6)

\begin{tabular}{|c|c|c|c|c|c|c|c|c|c|c|c|}
\hline Varies & Varies & $\mathrm{P}(\mathrm{T} 2-\mathrm{T} 3)$ & $\mathrm{P}(\mathrm{T} 2-\mathrm{T} 4)$ & $P(T 2-T 5)$ & $P(T 2-T 6)$ & P(T3-T4) & P(T3-T5) & P(T3-T6) & P(T4-T5) & $\mathrm{P}(\mathrm{T} 4-\mathrm{T} 6)$ & P(T5- \\
\hline \multirow[t]{2}{*}{ functional limitation } & Surgery-first group & $0.024^{*}$ & $0.000^{* * *}$ & $0.000^{* * *}$ & $0.000^{* * *}$ & 0.658 & 0.725 & 0.687 & 0.801 & 0.831 & 0.882 \\
\hline & Orthodontic-first group & 0.824 & $0.000^{* * *}$ & $0.000^{* * *}$ & $0.000^{* * *}$ & $0.000^{* * *}$ & $0.000^{* * *}$ & $0.000^{* * *}$ & 0.654 & 0.702 & 0.783 \\
\hline \multirow[t]{2}{*}{ physical pain } & Surgery-first group & $0.048^{*}$ & $0.004^{* *}$ & $0.000^{* * *}$ & $0.000^{* * *}$ & 0.624 & 0.742 & $0.049^{*}$ & 0.903 & 0.921 & 0.920 \\
\hline & Orthodontic-first group & 0.768 & $0.000^{* * *}$ & $0.000^{* * *}$ & $0.000^{* * *}$ & $0.000^{* * *}$ & $0.000^{* * *}$ & $0.000^{* * *}$ & 0.726 & 0.824 & 0.931 \\
\hline \multirow[t]{2}{*}{ psychological discomfort } & Surgery-first group & $0.003^{* *}$ & $0.000^{* * *}$ & $0.000^{* * *}$ & $0.000^{* * *}$ & $0.000^{* * *}$ & $0.000^{* * *}$ & $0.000^{* * *}$ & 0.869 & 0.902 & 0.965 \\
\hline & Orthodontic-first group & 0.857 & $0.000^{* * *}$ & $0.000^{* * *}$ & $0.000^{* * *}$ & $0.000^{* * *}$ & $0.000^{* * *}$ & $0.000^{* * *}$ & 0.867 & 0.798 & 0.804 \\
\hline \multirow[t]{2}{*}{ physical disability } & Surg & 0.00 & 0.00 & $0.000^{* * *}$ & $0.000^{* * *}$ & 0.687 & $0.000^{* * *}$ & $0.000^{* * *}$ & 0.145 & 0.263 & 37 \\
\hline & Orthodontic-first group & 0.867 & $0.000^{* * *}$ & $0.000^{* * *}$ & $0.000^{* * *}$ & $0.000^{* * *}$ & $0.000^{* * *}$ & $0.000^{* * *}$ & $0.042^{*}$ & $0.032^{*}$ & 0.903 \\
\hline \multirow[t]{2}{*}{ psychological disability } & Surgery-first group & $0.004^{* *}$ & $0.000^{* * *}$ & $0.000^{* * *}$ & $0.000^{* * *}$ & $0.000^{* * *}$ & $0.000^{* * *}$ & $0.000^{* * *}$ & $0.035^{*}$ & $0.038^{*}$ & 0.867 \\
\hline & Orthodontic-first group & 0.864 & $0.000^{* * *}$ & $0.000^{* * *}$ & $0.000^{* * *}$ & $0.000^{* * *}$ & $0.000^{* * *}$ & $0.000^{* * *}$ & $0.024^{*}$ & $0.034^{*}$ & 0.902 \\
\hline \multirow[t]{2}{*}{ social disability } & Surgery-first & $0.000^{* * *}$ & $0.000^{* * *}$ & $0.000^{* * *}$ & $0.000^{* * *}$ & 0.214 & $0.000^{* * *}$ & $0.000^{* * *}$ & $0.042^{*}$ & $0.038^{*}$ & 0.824 \\
\hline & Orthodontic-first group & 0.875 & $0.000^{* * *}$ & $0.000^{* * *}$ & $0.000^{* * *}$ & $0.000^{* * *}$ & $0.000^{* * *}$ & $0.000^{* * *}$ & $0.042^{*}$ & $0.024^{*}$ & 0.897 \\
\hline \multirow[t]{2}{*}{ handicaps } & Surgery-first group & 0.321 & $0.000^{* * *}$ & $0.000^{* * *}$ & $0.000^{* * *}$ & $0.000^{* * *}$ & $0.000^{* * *}$ & $0.000^{* * *}$ & 0.365 & 0.487 & 0.867 \\
\hline & Orthodontic-first group & 0.806 & $0.000^{* * *}$ & $0.000^{* * *}$ & $0.000^{* * *}$ & $0.000^{* * *}$ & $0.000^{* * *}$ & $0.000^{* * *}$ & $0.035^{*}$ & $0.008^{* *}$ & 0.421 \\
\hline \multirow[t]{2}{*}{ Totalscores } & Surg & $0.000^{* * *}$ & $0.000^{* * *}$ & $0.000^{* * *}$ & $0.000^{* * *}$ & $0.000^{* * *}$ & $0.000^{* * *}$ & $0.000^{* * *}$ & $0.042^{*}$ & $0.031^{*}$ & 0.682 \\
\hline & Orthodontic-first group & 0.654 & $0.000^{* * *}$ & $0.000^{* * *}$ & $0.000^{* * *}$ & $0.000^{* * *}$ & $0.000^{* * *}$ & $0.000^{* * *}$ & $0.000^{* * *}$ & $0.000^{* * *}$ & 0.725 \\
\hline
\end{tabular}

${ }^{*} P<0.05 ;{ }^{* *} P<0.01 ; * * * P<0.001$

Table 5 Comparisons of quality of life scores at the different time points for the surgery-first group and orthodontic-first group at 1 month, 6 month and 12 month after orthognathic surgery

\begin{tabular}{|c|c|c|c|c|c|c|c|c|c|}
\hline \multirow[t]{3}{*}{ Varies } & \multicolumn{2}{|l|}{ Postoperative } & \multirow[t]{3}{*}{$P$} & \multirow{2}{*}{\multicolumn{2}{|c|}{$\frac{\text { Postoperative }}{6 \text { month }}$}} & \multirow[t]{3}{*}{$P$} & \multirow{2}{*}{\multicolumn{2}{|c|}{$\frac{\text { Postoperative }}{12 \text { month }}$}} & \multirow[t]{3}{*}{$P$} \\
\hline & \multicolumn{2}{|l|}{1 month } & & & & & & & \\
\hline & $\begin{array}{l}\text { Surgery-first } \\
\text { group(T2) }\end{array}$ & $\begin{array}{l}\text { Orthodontic-first } \\
\text { group(T4) }\end{array}$ & & $\begin{array}{l}\text { Surgery-first } \\
\text { group(T3) }\end{array}$ & $\begin{array}{l}\text { Orthodontic-first } \\
\text { group(T5) }\end{array}$ & & $\begin{array}{l}\text { Surgery-first } \\
\text { group(T4) }\end{array}$ & $\begin{array}{l}\text { Orthodontic-first } \\
\text { group(T6) }\end{array}$ & \\
\hline \multirow{2}{*}{$\begin{array}{l}\text { functional } \\
\text { limitation }\end{array}$} & 5.22 & 5.42 & \multirow[t]{2}{*}{ NS } & 2.39 & 1.98 & \multirow[t]{2}{*}{ NS } & 1.99 & 1.92 & \multirow[t]{2}{*}{ NS } \\
\hline & (1.88) & (1.98) & & $(1.24)$ & (1.68) & & $(1.76)$ & $(1.56)$ & \\
\hline \multirow[t]{2}{*}{ physical pain } & 3.22 & 3.51 & \multirow[t]{2}{*}{ NS } & 2.34 & 1.89 & \multirow[t]{2}{*}{ NS } & 1.59 & 1.75 & \multirow[t]{2}{*}{ NS } \\
\hline & $(1.55)$ & $(1.72)$ & & $(1.24)$ & (1.28) & & $(0.67)$ & $(1.22)$ & \\
\hline \multirow{2}{*}{$\begin{array}{l}\text { psychological } \\
\text { discomfort }\end{array}$} & 4.29 & 4.82 & \multirow[t]{2}{*}{ NS } & 2.22 & 2.82 & \multirow[t]{2}{*}{ NS } & 0.56 & 0.92 & \multirow[t]{2}{*}{ NS } \\
\hline & $(1.22)$ & $(2.29)$ & & $(1.12)$ & $(1.31)$ & & $(1.10)$ & $(1.26)$ & \\
\hline \multirow[t]{2}{*}{ physical disability } & 4.15 & 4.42 & \multirow[t]{2}{*}{ NS } & 1.64 & 2.41 & \multirow[t]{2}{*}{ NS } & 1.34 & 1.49 & \multirow[t]{2}{*}{ NS } \\
\hline & $(1.72)$ & $(1.75)$ & & $(1.08)$ & (1.13) & & $(1.22)$ & $(0.91)$ & \\
\hline \multirow{2}{*}{$\begin{array}{l}\text { psychological } \\
\text { disability }\end{array}$} & 3.28 & 3.72 & \multirow[t]{2}{*}{ NS } & 1.64 & 1.83 & \multirow[t]{2}{*}{ NS } & 0.42 & 0.88 & \multirow[t]{2}{*}{ NS } \\
\hline & $(1.76)$ & (1.58) & & (1.08) & $(1.15)$ & & $(0.21)$ & $(1.13)$ & \\
\hline \multirow[t]{2}{*}{ social disability } & 4.26 & 4.52 & \multirow[t]{2}{*}{ NS } & 1.24 & 1.64 & \multirow[t]{2}{*}{ NS } & 0.62 & 0.98 & \multirow[t]{2}{*}{ NS } \\
\hline & (1.98) & $(2.56)$ & & $(1.08)$ & $(1.18)$ & & $(0.35)$ & $(1.12)$ & \\
\hline \multirow[t]{2}{*}{ handicaps } & 2.18 & 2.42 & \multirow[t]{2}{*}{ NS } & 1.52 & 1.94 & \multirow[t]{2}{*}{ NS } & 0.38 & 0.78 & NS \\
\hline & $(1.81)$ & $(2.12)$ & & $(1.75)$ & $(1.36)$ & & $(0.26)$ & $(1.12)$ & \\
\hline Total scores & 27.72 & 28.86 & NS & 13.94 & 15.61 & NS & 6.90 & 8.68 & NS \\
\hline & $(3.26)$ & (3.83) & & $(2.13)$ & $(2.49)$ & & (1.39) & (1.65) & \\
\hline
\end{tabular}


ranged between $70 \%$ [32] and $87 \%$ [33], and which was also higher than that reported among pre-treatment and no-treatment control groups [34]. Hence, positive changes occurred in the personality profiles of patients. There was an obvious improvement in self-confidence in $67.5 \%$ of patients as a result of an improved appearance and an improved chewing function.

The results of the present study showed a highly significant degree of overall improvement in patients'quality of life after orthognathic surgery in two group, others found the similar resluts [35-37]. However, we noticed that the quality of life scores was deteriorated before orthognathic in orthodontic-first group. This results indicated that the dental decompensation during preorthodontics could worsen the facial deformity, which has been perceived as the most stressful period of overall treatment by the patients [38] Esperao [39] found the similar results. It reminded us that we may need tell patients that he may experience short-term quality of life deterioration before orthognathic surgery.

We were very pleased to find that all the domain of quality of life scores were consistently lower in the surgery-first groups compared to the orthodontic-first group, although there were no significant statistical differences when patients received orthognathic surgery. The reason may be that surgery -first treatment could improve OHRQoL immediately and lead to better satisfactory compare to orthodontic-first group. It was important to doctors, because we not only gain perfect treatment results but also improve patients'quality of life and satisfaction.

The limitations of the study was small sample,we only included 50 patients which may weak the evidence of this study. Secondly, we did not objectively quantify the quality of orthodontic outcome. Although patient satisfaction is high, it has been noted by the orthodontists treating some of these patients that there is an urge to remove the orthodontic appliances very soon after orthognathic surgery. In future research we need including treatment results when assess the quality of life changes. Additionally, because of inclusion criteria of this article was limited to BSSRO, we think if two-jaw surgery was included in the study, the results will be different. In present, because of raised patient's requests, two-jaw surgery increases. Thus, future research need includ more two-jaw surgery patients.

\section{Conclusions}

Surgery-first treatment could significant reduce treatment during and no deterioration stage of quality of life score which lead to better satisfactory compare to orthodontic-first group. However, some of limitations we need take caution, such as small sample, no two-jaw surgery patients were included. In future we need conduct a more larger sample randomized control trail to investigate the oral health-related quality of life in Chinese orthognathic surgery patients.

\section{Competing interests}

The authors declare that they have no competing interests.

\section{Authors' contributions}

ZY,SH and WC designed the study, gathered the information, performed the statistical analysis and wrote the first draft of the manuscript. ZN designed the form for data gathering and supervised the statistical analysis. All authors read and approved the final manuscript.

\section{Author details}

${ }^{1}$ Department of Prosthodontics, Hospital of Stomatology, Wenzhou Medical University, Wenzhou, China. ${ }^{2}$ Department of Orthodontics, Hospital of Stomatology, Wenzhou Medical University, 113 West College Road, 325000 Wenzhou, China.

Received: 24 November 2015 Accepted: 23 December 2015 Published online: 05 January 2016

References

1. Ong MAH. Spectrum of dento-facial deformities: a retrospective survey. Ann Acad Med Singapore. 2004;33:239-42.

2. Cunningham SJ, Garratt AM, Hunt NP. Development of a condition-specific quality of life measure for patients with dentofacial deformity: II. Validity and responsiveness testing. Community Dent Oral Epidemiol. 2002;30:81-90.

3. Sarwer DB, Bartlett SP, Whitaker LA, Paige KT, Pertschuk MJ, Wadden TA. Adult psychological functioning of individuals born with craniofacial anomalies. Plast Reconstr Surg. 1999;103:412-8.

4. Versnel SL, Duivenvoorden HJ, Passchier J, Mathijssen IMJ. Satisfaction with facial appearance and its determinants in adults with severe congenital facial disfigurement: a case-referent study. J Plast Reconstr Aesthet Surg. In press.

5. Ahmed B, Gilthorpe MS, Bedi R. Agreement between normative and perceived orthodontic need amongst deprived multiethnic schoolchildren in London. Clin Orthod Res. 2001:4:65-71.

6. Hunt O, Hepper P, Johnston C, Stevenson M, Burden D. The aesthetic component of the index of orthodontic treatment need validated against lay opinion. Eur J Orthod. 2002;24:53-9.

7. Kiyak HA, Reichmuth M. Body image issues in dental medicine. In: Cash TF, Pruzinsky T, editors. Body image: a handbook of theory, research and clinical practice. New York: Guilford; 2002. p. 342-50.

8. Bellucci CC, Kapp-Simon KA. Psychological considerations in orthognathic surgery. Clin Plast Surg. 2007;34:e11-6.

9. Cunningham SJ, Hunt NP. Quality of life and its importance in orthodontics. J Orthod. 2001;28:152-8.

10. Bennett ME, Phillips CL. Assessment of health-related quality of life for patients with severe skeletal disharmony: a review of the issues. Int J Adult Orthod Orthognath Surg. 1999;14:65-75.

11. Han CW, Yajima Y, Nakajima K, Lee EJ, Meguro M, Kohzuki M. Construct validity of the Frenchay activities index for community-dwelling elderly in Japan. Tohoku J Exp Med. 2006;210:99-107.

12. Baek SH, Ahn HW, Kwon YH, Choi JY. Surgery-first approach in skeletal Class III malocclusion treated with 2- jaw surgery: evaluation of surgical movement and postoperative orthodontic treatment. J Craniofac Surg. 2010;21:332-8.

13. Ko EW, Hsu SS, Hsieh HY, Wang YC, Huang CS, Chen YR. Comparison of progressive cephalometric changes and postsurgical stability of skeletal Class III correction with and without presurgical orthodontic treatment. J Oral Maxillofac Surg. 2011;69:1469-77.

14. Liou EJ, Chen PH, Wang YC, Yu CC, Huang CS, Chen YR. Surgery-first accelerated orthognathic surgery: orthodontic guidelines and setup for model surgery. J Oral Maxillofac Surg. 2011;69:771-80.

15. Liao YF, Chiu YT, Huang CS, Ko EW, Chen YR. Presurgical orthodontics versus no presurgical orthodontics: treatment outcome of surgical-orthodontic correction for skeletal Class III open bite. Plast Reconstr Surg. 2010;126:2074-83.

16. Modig M, Andersson L, Wardh I. Patients' perception of improvement after orthognathic surgery: pilot study. Br J Oral Maxillofac Surg. 2006;44:24-7. 
17. Motegi E, Hatch JP, Rugh JD. Health related quality of life and psychosocial function 5 years after orthognathic surgery. Am J Orthod Dentofacial Orthop. 2003;124:138-43.

18. NicodemoD, PereiraMD, FerreiraLM. Effect of orthognathic surgery for class III correction asmeasured by SF-36. Int Joral Maxillofac Surg 2007: 1231-1234.

19. Murphy C, Kearns G, Allen PF. The clinical relevance of orthognathic surgery on quality of life. Int J Oral Maxillofac Surg. 2011;40:926-30.

20. Leao A, Sheiham A. Relation between clinical dental status and subjective impacts on daily living. J Dent Res. 1995;74:1408-13.

21. Euro Qol Group. EuroQol-a new facility for the measurement of healthrelated quality of life. Health Policy. 1990;16:199-208.

22. Proffit WR, Miguel JA. The duration and sequencing of surgical-orthodontic treatment. Int J Adult Orthodon Orthognath Surg. 1995;10:35-42.

23. Slavnic $S$, Marcusson A. Duration of orthodontic treatment in conjunction with orthognathic surgery. Swed Dent J. 2010;34:159-66.

24. O'Brien K, Wright J, Conboy F, Appelbe P, Bearn D, Caldwell S, et al. Prospective, multi-center study of the effectiveness of orthodontic/ orthognathic surgery care in the United Kingdom. Am J Orthod Dentofacial Orthop. 2009;135:709-14.

25. Diaz PM, Garcia RG, Gias LN, Aguirre-Jaime A, Perez JS, de la Plata MM, et al Time used for orthodontic surgical treatment of dentofacial deformities in white patients. J Oral Maxillofac Surg. 2010;68:88-92.

26. Huang CS, Hsu SS, Chen YR. Systematic review of the surgery-first approach in orthognathic surgery. Biomed J. 2014;37:184-90.

27. Yu CC, Chen PH, Liou EJ, Huang CS, Chen YR. A Surgery-first approach in surgical-orthodontic treatment of mandibular prognathism - a case report. Chang Gung Med J. 2010;33:699-705.

28. Nagasaka H, Sugawara J, Kawamura H, Nanda R. "Surgery first" skeletal Class III correction using the skeletal anchorage system. J Clin Orthod. 2009;43:97-105.

29. Villegas C, Uribe F, Sugawara J, Nanda R. Expedited correction of significant dentofacial asymmetry using a "surgery first" approach. J Clin Orthod. 2010; 44:97-103.

30. Leao A. The Development of Measures of Dental Impacts on Daily Living [PhD thesis]. London, UK: London University; 1993.

31. Al-Omiri MK. Tooth Wear Impact on Daily Living [PhD thesis]. Belfast, UK: Queen's University Belfast; 2002.

32. Türker N, Varol A, Ogel K, Basa S. Perceptions of preoperative expectations and postoperative outcomes from orthognathic surgery: part l: Turkish female patients. Int J Oral Maxillofac Surg. 2008;37:710-5.

33. De Clercq CA, Neyt LF, Mommaerts MY, Abeloos JS. Orthognathic surgery: patients' subjective findings with focus on the temporomandibular joint. J Cranio-maxillo-facial Surg. 1998;26:29-34.

34. Lazaridou-Terzoudi T, Kiyak HA, Moore R, Athanasiou AE, Melsen B. Longterm assessment of psychologic outcomes of orthognathic surgery. J Oral Maxillofac Surg. 2003;61:545-52.

35. Tabrizi R, Rezaii A, Golkari A, Ahrari F. The impact of orthognathic surgery on oral health-related quality of life. J Dent Mater Technol. 2014;3:23-7.

36. Soh CL, Narayanan V. Quality of life assessment in patients with dentofacia deformity undergoing orthognathic surgery-a systematic review. Int J Oral Maxillofac Surg. 2013;42:974-80.

37. Kavin T, Jagadesan AG, Venkataraman SS. Changes in quality of life and impact on patients' perception of esthetics after orthognathic surgery. J Pharm Bioallied Sci. 2012;4:S290-3.

38. Hernndez-Alfaro F, Guijarro-Martnez R, Molina Coral A, Bada-Escriche C. "Surgery first" in bimaxillary orthognathic surgery. J Oral Maxillofac Surg. 2011;69:201-7.

39. Esperão PT, de Oliveira BH, de Oliveira Almeida MA, Kiyak HA, Miquel JA Oral health-related quality of life in orthognathic surgery patients. Am J Orthod Dentofacial Orthop. 2010;137:790-5.

\section{Submit your next manuscript to BioMed Central and we will help you at every step:}

- We accept pre-submission inquiries

- Our selector tool helps you to find the most relevant journal

- We provide round the clock customer support

- Convenient online submission

- Thorough peer review

- Inclusion in PubMed and all major indexing services

- Maximum visibility for your research

Submit your manuscript at www.biomedcentral.com/submit

C) Biomed Central 